Márcio Luís Duarte ${ }^{1}$, Ralff Mallmann², Simone Botelho Alvarenga ${ }^{3}$, Lucas Ribeiro dos Santos ${ }^{4}$, José Luiz Masson de Almeida Prado5[D, Luiz Carlos Donoso Scoppetta ${ }^{6}$ (i)

${ }^{1}$ WEBIMAGEM Telerradiologia, São Paulo, Brazil

${ }^{2}$ Mercês Clinical Centre, Curitiba, Paraná, Brazil

${ }^{3}$ Axial Diagnostic Medicine, Belo Horizonte, Minas Gerais, Brazil

${ }^{4}$ Faculty of Medical Sciences of Santos, UNILUS, Santos, São Paulo, Brazil

${ }^{5}$ WEBIMAGEM Telerradiologia, São Paulo/SP, Brazil

${ }^{6}$ São Camilo Hospital, São Paulo, São Paulo, Brazil

\title{
Lower limb bilateral pyomyositis in a diabetic patient: case report and literature review
}

\begin{abstract}
Pyomiositis is a bacterial infection of skeletal muscle tissue occurring mostly in immunocompromised patients, including diabetics. The main agents are Gram-positive cocci. We report a case of a 42 years old, male, diabetic, who presented the emergency room referring pain in thighs and inability to flex his legs. We performed a magnetic resonance which revealed extensive purulent collections in both lower limbs, thus confirming clinical suspicion, and allowing proper antibiotic treatment. In this case, we showed that imaging tests facilitate early diagnosis and treatment through direct location of the lesions, and guides invasive procedures such as biopsy and abscess aspiration when needed. (Clin Diabetol 2020; 9; 2: 134-137)
\end{abstract}

Key words: pyomyositis, diabetes mellitus type 2, magnetic resonance imaging

Address for correspondence:

Márcio Luis Duarte, MD, MSc

WEBIMAGEM Telerradiologia

Avenida Marquês de São Vicente 446, São Paulo

São Paulo, Brazil

Zip Code: 04104-020

e-mail: marcioluisduarte@gmail.com

Clinical Diabetology 2020, 9, 2, 134-137

DOI: $10.5603 /$ DK.2020.0003

Received: 03.09.2019

Accepted: 17.12.2019

\section{Introduction}

Scriba, in 1885, reported the first case of pyomyositis [1], as a primary acute bacterial infection of skeletal muscle's tissue [1, 2]; it occurs mainly in immunocompromised patients [3], malnurished [4] or, exceptionally, in immunocompetent patients in tropical areas [3]. In fact, it was originally described as a tropical disease and is now being seen, with increasing frequency, in temperate regions; pyomyositis holds a mortality rate of about $10 \%$ and may also be responsible for complications such as abscesses, septicemia, and shock [2].

The pathogenesis of primary pyomyositis remains unclear, although trauma and immune impairment can be predisposing factors [2]. It affects individuals, preferably during the first and second decades of life, and is more prevalent in men [1]; patients over 30 years of age usually have associated comorbidities [5], and the most described in the literature are human immunodeficiency virus infection [6], diabetes mellitus, malignant tumors, liver cirrhosis, renal failure, transplantation and use of immunosuppressive drugs, and intravenous drug abuse [1, 3]. Its initial signs and symptoms are usually subtle and can be easily overlooked or misdiagnosed as a more benign condition [2], specially erysipelas.

Pyomyositis can affect any muscle group, infecting by contiguity or hematogenous spread $[1,5]$. The main agents are Gram-positive cocci [3], and the most common etiologic agent is Staphylococcus aureus, in up to $85-95 \%$ of the cases $[1,2,5]$ - with increasing frequency of resistance to methicillin (MRSA) [2]. 

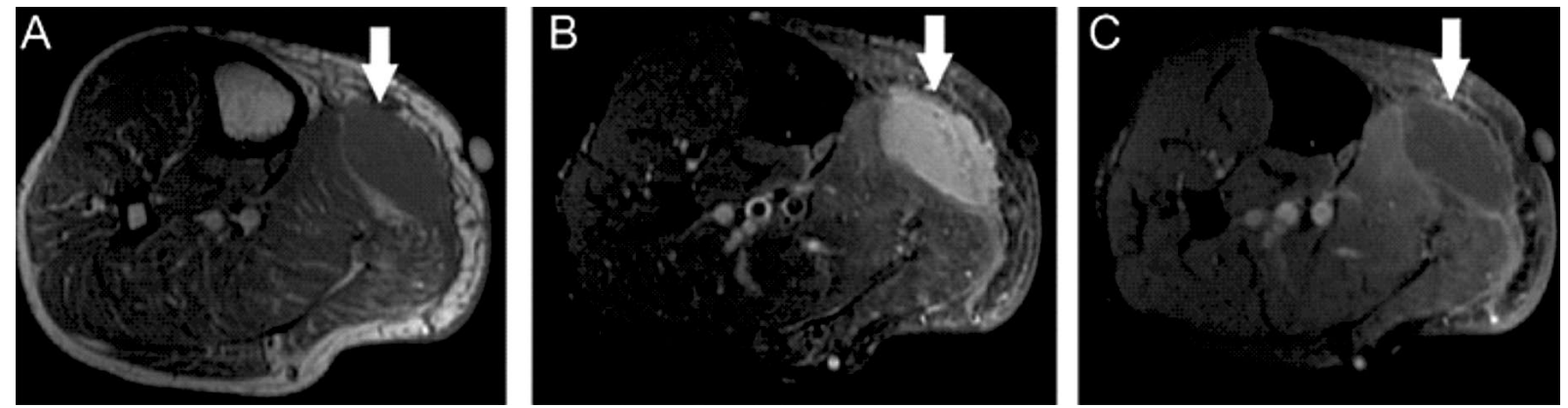

Figure 1. MRI of the right leg in the axial section T1-weighted sequence in A, T2 STIR in B and T1 sequence with contrast in C demonstrating a collection between the musculature and subcutaneous tissue with peripheral contrast enhancement (white arrows)
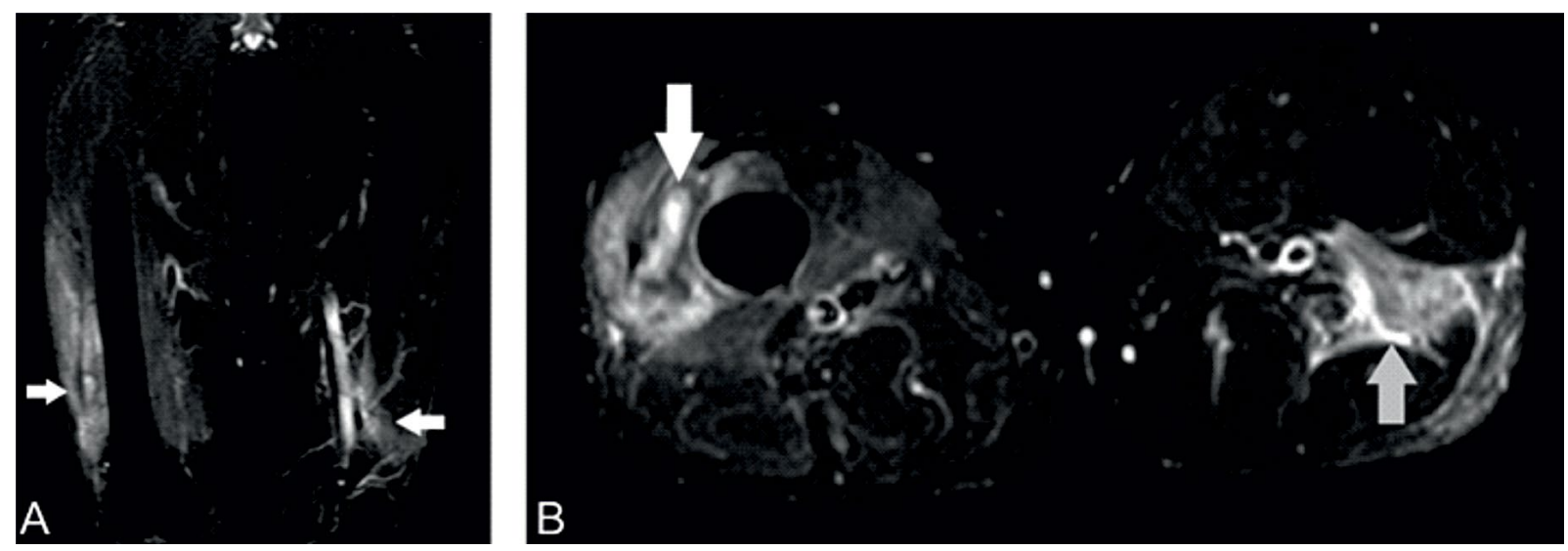

Figure 2. MRI of the thighs in the coronal section T2 STIR in A and axial section in B, demonstrating swelling of the right vastus lateralis and left femoral biceps muscles (white arrows in A) with collection in the right vastus medius muscle (white arrow in B), edema of the other muscles bellies with subfascial and peripheral fluid (gray arrow in B)

\section{Case report}

The patient is a 42 years old, Brazilian male, security agent, referred to the emergency department complaining of a acute pain of severe intensity on both thighs, restraining flexion of both lower limbs for the last seven days. He reported that a less severe pain had been present for over two months, and, one month earlier, he was hospitalized for 15 days due to erysipelas in his right leg - received analgesics without improvement at that time. He denied smoking or trauma, reporting, just social drinking; as a family background his father was diabetic and his mother had arterial hypertension. Physical examination of the patient showed a hyperemic, swollen and painful right leg, especially in the calf; his body temperature was $38.2^{\circ} \mathrm{C}$ pulse of $98 \mathrm{ppm}$ and blood pressure of 150 over $80 \mathrm{~mm} \mathrm{Hg}$. Laboratory workup showed increased leukocyte count $\left(18,452 \mathrm{hg} / \mathrm{mm}^{3}\right)$ and elevated c-reactive protein $15 \mathrm{mg} / \mathrm{dL}$. Throughout this hospitalization, he was also diagnosed with type 2 diabetes mellitus and systemic arterial hypertension, having started treatment since then, and had good glucose control throughout hospitalization with metformin.

During the first hospitalization, a magnetic resonance image (MRI) of the right leg was performed (Figure 1 - description on the legend), but it might had been neglected. Given the progression of symptoms and severity of the clinical setting, a new MRI of both thighs was performed (Figure 2 - description on the legend), which showed purulent collection affecting both legs, rising the diagnosis of bilateral pyomyositis stage 2. Patient started to receive ceftriaxone plus teicoplanin empirically, and after 2 weeks was discharged with complete resolution.

\section{Discussion}

The diagnosis of pyomyositis can be challenging due to the nonspecific clinical signs at onset [2]. It may be confused with bone or joint infection processes, thrombophlebitis, panniculitis or systemic diseases [2]. The differential diagnosis includes muscle trauma, deep vein thrombosis, osteomyelitis, cellulitis, septic arthritis, and malignant tumors [2]. 
In the case of diabetic patients, it appears that muscular and circulatory disorders which, together with granulocyte dysfunction and decreased cellular immunity, increase the risk of developing pyomyositis [4]. Laboratory findings are nonspecific and the blood count may show leukopenia or leukocytosis with left deviation, depending on the etiologic agent and the patient immune response $[1,4]$. There may be increased inflammatory markers and generally does not affect kidney function $[3,5]$. Blood cultures are positive in less than $40 \%$ of patients and secretion cultures are positive in only $21-41 \%$ of cases [6].

Usually, it only affects one muscle group, but in $11 \%$ to $43 \%$ of patients may have widespread outbreaks in various muscles, being quadriceps muscle the most affected, followed by the gluteal and iliopsoas muscles [7]; iliopsoas pyomyositis occurs more commonly secondary to gastrointestinal or urinary tract infections [7].

Pyomyositis presents three evolutionary stages: invasive, purulent and late. The invasive stage is characterized by insidious onset, where only about $2 \%$ of affected patients seek medical support. The purulent stage occurs about 10-21 days after symptom onset and is characterized by the abscess location and, at this stage, about $90 \%$ of patients seek medical care due to the severity of the symptoms. If the disease is not identified and treated properly with antibiotics and/or surgical drainage, evolution occurs for the so-called late period associated with significant systemic involvement like septic embolization, septic shock, and even death [8]. Our patient had a bilateral phase 2 pyomiositis, due to an initial misdiagnosis of erysipelas, when he was still on initial phase.

Imaging tests such as ultrasound, computed tomography (CT) and magnetic resonance imaging (MRI) are useful to identify the number, size, extension, location of abscesses, and guide needle biopsy [9].

Ultrasound has high sensitivity for fluid collections detection; it may demonstrate initial muscle edema as a hypoechoic lesion with imprecise limits affecting one or more muscles that, with latter disease evolution, can lead to the formation of abscess [3].

CT scan shows enlargement and decreased attenuation of the affected muscle, with a blurring of surrounding fat. The involvement of a muscle group that is disproportionate to the involvement of the subcutaneous tissue helps distinguish myositis from primary cellulitis. Intramuscular collections can be observed and contrast material is used to help differentiate viable and necrotic muscles by demonstrating enhancement around the abscess [5, 7]. CT scan, as well as ultrasound, can help guide needle aspiration [3] and are also useful for surgical planning, with abscess drainage muscle followed by culture-guided antibiotics [5, 7].

MRI is the gold standard for the diagnosis by detecting early findings of muscles diffuse inflammation [5], and may also guide biopsy of the affected muscles [10]. MRI can show extension of infection and evaluates adjacent structures, such as joints, bone, and other surrounding soft tissues, differentiating pyomyositis from other differential diagnosis.

Treatment with antibiotics in conjunction with surgical drainage or suction, when applicable, is generally sufficient; prognosis is good with early diagnosis and appropriate treatment, depending on the underlying comorbities [11, 12]. Usually, one antibiotic with $S$. aureus coverage such as oxacillin, ciprofloxacin or cephalosporins is enough, but when dealing with immunocompromised patients, association of a amynoglicoside or even glycopeptides may be useful [5].

Untreated pyomyositis complications may include compartment syndrome, progression infection to septic arthritis, osteomyelitis and even death, which ranges between in up to $10 \%$ of the cases $[5,7]$. In the long term, patients may experience weakness, and muscle dysfunction [5, 7].

In conclusion, pyomyositis may have subtle symptoms that are easily misinterpreted as signs of a more common and benign infection. Imaging tests facilitate early diagnosis and treatment through the direct location of the lesions and, when it's necessary, assist invasive procedures such as biopsy and abscess aspiration. The early recognition of the disease allows an earlier treatment preventing the development of complications.

\section{Conflict of interest}

We state that the authors have no conflict of interest.

\section{REFERENCES}

1. Polizelli D, Geraldino G, Narvaes E, et al. Piomiosite associada ao diabetes mellitus e cirrose hepática. Rev Bras Reumatol. 2010; 50(4): 472-477, doi: 10.1590/s0482-50042010000400011.

2. Lemonick DM. Non-tropical pyomyositis caused by methicillin-resistant Staphylococcus aureus: an unusual cause of bilateral leg pain. J Emerg Med. 2012; 42(3): e55-e62, doi: 10.1016/j. jemermed.2008.12.020, indexed in Pubmed: 19327931.

3. Bureau NJ, Chhem RK, Cardinal E. Musculoskeletal infections: US manifestations. Radiographics. 1999; 19(6): 1585-1592, doi: 10.1148/radiographics.19.6.g99no061585, indexed in Pubmed: 10555676.

4. Scully RE, Mark EJ, McNelly WF. EbelingSH, Phillips LD. Case 291997: case records of the Massachusetts General Hospital. N Engl J Med. 1997; 337: 839-845.

5. Bickels J, Ben-Sira L, Kessler A, et al. Primary pyomyositis. J Bone Joint Surg Am. 2002; 84(12): 2277-2286, doi: 10.2106/00004623200212000-00024, indexed in Pubmed: 12473721. 
6. Christin L, Sarosi GA. Pyomyositis in North America: case reports and review. Clin Infect Dis. 1992; 15(4): 668-677, doi: 10.1093/ clind/15.4.668, indexed in Pubmed: 1420680.

7. Fayad LM, Carrino JA, Fishman EK. Musculoskeletal infection: role of CT in the emergency department. Radiographics. 2007; 27(6): 1723-1736, doi: 10.1148/rg.276075033, indexed in Pubmed: 18025514

8. Pereira F, Medeiros Y. Síndromes dolorosas em diabéticos por comprometimento de musculatura esquelética. Arq Bras Endocrinol Metab. 2006; 50(5): 957-962, doi: 10.1590/s000427302006000500020
9. Soler R, Rodríguez $E$, Aguilera $C$, et al. Magnetic resonance imaging of pyomyositis in 43 cases. Eur J Radiol. 2000; 35(1): 59-64, doi: 10.1016/s0720-048x(99)00108-4, indexed in Pubmed: 10930768.

10. Schweitzer ME, Fort J. Cost-effectiveness of MR imaging in evaluating polymyositis. AJR Am J Roentgenol. 1995; 165(6): 1469-1471, doi: 10.2214/ajr.165.6.7484589, indexed in Pubmed: 7484589.

11. Al-Tawfiq JA, Sarosi GA, Cushing HE. Pyomyositis in the acquired immunodeficiency syndrome. South Med J. 2000; 93(3): 330-334, indexed in Pubmed: 10728527.

12. Azevedo PS, Matsui M, Matsubara LS, et al. Piomiosite tropical: apresentações atípicas. Rev Soc Bras Med Trop. 2004; 37(3): 273-278. 